\title{
Optimal excitation for nonparametric identification of viscoelastic materials
}

\author{
Agnes Rensfelt and Torsten Söderström \\ Div. of Systems and Control, Dept. of Information Technology, \\ Uppsala University, Box 337, SE-751 05 Uppsala, Sweden.
}

June 27, 2006

\begin{abstract}
The problem of optimal excitation in nonparametric identification of viscoelastic materials is considered. The goal is to design the input spectrum in an optimal way, so that the average variance of the estimates is minimized. It is shown how the covariance matrix of the estimates can be expressed in terms of the input spectrum. This theory can also be used in order to identify the (unknown) excitation, used in a particular experiment, from measured strain data. Two scalar criteria connected to A- and D-optimal experiment design, are considered. The results indicate that the accuracy of the estimates can be greatly improved by applying an optimal input signal. Issues concerning the implementation of the achieved optimal input spectrum in live experiments are discussed briefly.
\end{abstract}

\section{Introduction}

Viscoelastic materials, such as plexiglass and other plastics, can today be found in a wide range of practical applications. In order to make efficient use of these materials, it is of interest to understand their behavior when used in an environment where the material is subjected to dynamic load. Such dynamic load could for example be vibrations from a motor, or stress put on to the structure through collision or impact.

A viscoelastic material is characterized by its frequency dependent complex modulus $E(\omega)$, that relates stress and strain in the material. In the frequency domain this relationship looks like

$$
\sigma(\omega)=E(\omega) \varepsilon(\omega),
$$


where $\sigma(\omega)$ and $\varepsilon(\omega)$ denotes the Fourier transformed stress and strain, respectively. Knowledge about the complex modulus is essential in understanding the materials behavior in a dynamic environment, and can be determined through different kinds of wave propagation experiments, as studied in [1], [10] and [23]. In order to get good quality estimates, the collected data should contain as much valuable information as possible, and design parameters that influence the information content must thus be chosen carefully. Examples of such parameters are the input excitation signal, the number of sensors used in the experiment, and the sensor locations. The procedure of determining optimal design parameters in an experiment is commonly referred to as optimal experiment design.

A solid theoretical basis for optimal experiment design is built in [3] and [20], and is widely employed in different areas of engineering. Some examples include sensor array signal processing [8] and robotics [13]. The problem of goal-oriented experiment design has also received a lot of attention, see for example [7], [11], [4] and [6]. In the control literature, a survey on the sensor location problem is presented in [12], while the input design problem is treated in [16]. A more recent discussion on input design can be found in [25]. A common procedure for optimal experiment design is to minimize some scalar function of the covariance matrix of the estimate. The design tools developed is therefore useful in a wide range of applications, where the covariance matrix can be expressed as a function of the design variable of interest.

This work will focus on the subject of optimal input signal for identification of viscoelastic materials. The subject of optimal sensor locations for this application has been studied in [18] and [21]. Previously, the preferred kind of input has been an strain pulse generated by impact from a steel hammer or an air gun, and the transient response following the pulse was then studied. However, in order to get good estimates it is important to give sufficient excitation to all frequencies considered in the identification, i.e. the input signal should contain enough energy at these frequencies. As an pulse tends to have the majority of its energy at low frequencies, and as identification of the complex modulus is frequently carried out for frequencies up to $15 \mathrm{kHz}$, this kind of excitation can be expected to be sub-optimal for identification in the higher frequency range. The question addressed here can thus be formulated as follows: if we have the same energy content as in the original pulse, and if we could freely control the frequency distribution of this energy, how would the optimal spectrum of the input signal look?

The paper is organized as follows. In the next section the identification experiments are described, followed by the modeling of the system in Section 3 and identification in Section 4. The optimal experiment design is 


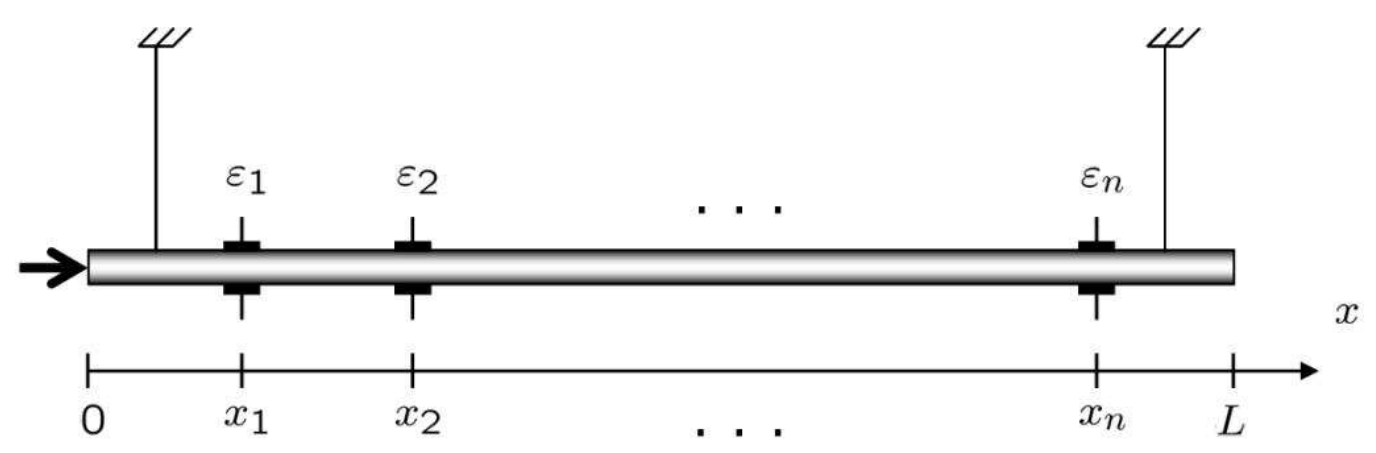

Figure 1: Experimental setup

described in Section 5, and the results are analyzed in Section 6. Finally, conclusions are drawn in Section 7.

\section{Experimental setups}

In this work, two kinds of wave propagation experiments will be studied. In one, longitudinal wave propagation is used, and the setup is shown in Figure 1. Here a slender bar of length $L$ is suspended horizontally with thin wires. The bar is then axially excited, giving rise to longitudinal waves traveling back and forth in the bar. The strains caused by the wave propagation are measured at $n$ different sections located at $\left\{x_{i}\right\}_{i=1}^{n}$, at $N$ discrete time instances. In order to avoid aliasing, the analog strain signals are passed through a low-pass anti-aliasing filter before sampling.

The second experiment use flexural wave propagation, and the setup is shown in Figure 2. Here a beam of length $L$ is mounted vertically, and is then excited laterally to give rise to flexural waves traveling along the beam. As in the longitudinal case, the associated strain data is collected at $n$ sections $\left\{x_{i}\right\}_{i=1}^{n}$, at $N$ discrete time instances. Flexural wave propagation experiments are often useful when the frequencies of interest are between $10 \mathrm{~Hz}$ and 1 $\mathrm{kHz}$. With longitudinal wave propagation on the other hand, it is possible to achieve estimates well above $1 \mathrm{kHz}$. Since longitudinal wave experiments are concerned with one dimensional wave propagation, the limiting factor instead is that we can only consider frequencies for which the wavelengths are much larger than the diameter of the bar. For these frequencies approximate $1 \mathrm{D}$ conditions will prevail in the setup. A common requirement is that the wavelengths must be at least ten times the diameter of the bar. Similarly a lower limit on useful frequencies can be estimated from the requirement that there must be a sufficient strain variation between the outermost sections $x_{1}$ 


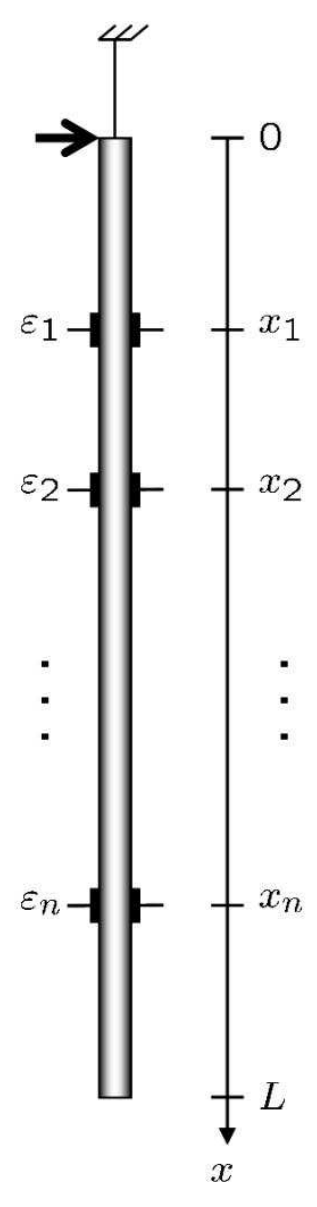

Figure 2: Experimental setup

and $x_{n}$, or that the wavelength must be shorter than 10 times the distance between these sections.

The identification is carried out in the frequency domain, and the recorded signals are therefor transformed using the Discrete Fourier Transform (DFT)

$$
\hat{\varepsilon}\left(\omega_{k}\right)=\frac{1}{\sqrt{N}} \sum_{n=0}^{N-1} \varepsilon(n) \mathrm{e}^{-\mathrm{i} \frac{2 \pi}{N} n k}
$$

for

$$
\omega_{k}=\left\{\begin{array}{ll}
\frac{2 \pi k}{N T}, & 0 \leq k \leq \frac{N}{2} \\
\frac{2 \pi(k-N)}{N T}, & \frac{N}{2}<k<N
\end{array} .\right.
$$

Since the measured signal is real-valued, it is sufficient to consider only the positive frequencies, i.e. only

$$
\omega_{k}=\frac{2 \pi k}{N T}, \quad 0<k<\frac{N}{2}
$$


will be considered in this study.

\section{Modeling}

The wave propagation in the previously described experiments can be modeled in a state-space framework. Note that in the following, all state variables are in the frequency domain and that the independent variable is the position $x$ along the center line of the bar/beam.

For an appropriate choice of state variables, the state vector $\mathbf{s}(x, \omega)$ will follow a system of first-order ordinary differential equations, see [9] or [24]

$$
\frac{d \mathbf{s}(x, \omega)}{d x}=\mathbf{R}(\omega) \mathbf{s}(x, \omega) .
$$

Note that the state vector and the matrix $\mathbf{R}(\omega)$ will be defined differently for the different experiments. These will be described in detail in the following subsections. Also note that the strain at section $x$ and frequency $\omega$ will be a linear combination of the elements in the state vector $\mathbf{s}(x, \omega)$, i.e.

$$
\varepsilon(x, \omega)=\mathbf{c}_{0}^{T}(\omega) \mathbf{s}(x, \omega) .
$$

The general solution to (5) is given by

$$
\begin{aligned}
\mathbf{s}(x, \omega)=\mathbf{c}_{1}^{-}(\omega) \mathrm{e}^{\gamma_{1}(\omega) x} & +\mathbf{c}_{1}^{+}(\omega) \mathrm{e}^{-\gamma_{1}(\omega) x}+\cdots \\
\cdots & +\mathbf{c}_{p}^{-}(\omega) \mathrm{e}^{\gamma_{p}(\omega) x}+\mathbf{c}_{p}^{+}(\omega) \mathrm{e}^{-\gamma_{p}(\omega) x},
\end{aligned}
$$

where $\left\{ \pm \gamma_{i}(\omega)\right\}_{i=1}^{p}$ are the eigenvalues of $\mathbf{R}(\omega)$, and are commonly referred to as the wave propagation functions. Here $\left\{-\gamma_{i}(\omega)\right\}_{i=1}^{p}$ correspond to the wave propagation in the positive $x$ direction of the bar/beam, and $\left\{\gamma_{i}(\omega)\right\}_{i=1}^{p}$ represent the reflected wave in the negative $x$ direction. The vectors $\left\{c_{i}^{ \pm}(\omega)\right\}_{i=1}^{p}$ are amplitudes of the associated waves at $x=0$. These vectors depend on various boundary condition, see Appendix A.

In the following subsections the state-space realizations of both the longitudinal and the flexural wave propagation models will be described.

\subsection{Longitudinal waves}

Consider a linearly viscoelastic, homogenous bar of length $L$, cross sectional area $A$, and density $\rho$. For longitudinal waves, the wave propagation at section $x$ can be described by the normal force $N(x, t)$ and the particle velocity $\dot{u}(x, t)$ at this section. This gives the frequency domain state vector

$$
\mathbf{s}(x, \omega)=[N(x, \omega) \quad \dot{u}(x, \omega)]^{T} .
$$


Further, the matrix $\mathbf{R}(\omega)$ in (5) can be defined as

$$
\mathbf{R}(\omega)=\left[\begin{array}{cc}
0 & \mathrm{i} \omega \rho A \\
\frac{\mathrm{i} \omega}{E(\omega) A} & 0
\end{array}\right]
$$

see [24] for details. This matrix has only two eigenvalues and the general solution (7) will thus be on the form

$$
\mathbf{s}(x, \omega)=\mathbf{c}^{-}(\omega) \mathrm{e}^{\gamma(\omega) x}+\mathbf{c}^{+}(\omega) \mathrm{e}^{-\gamma(\omega) x} .
$$

Also note that the relationship between the wave propagation function $\gamma(\omega)$ and the complex modulus $E(\omega)$ is

$$
\gamma^{2}(\omega)=-\frac{\rho \omega^{2}}{E(\omega)}
$$

For longitudinal waves, the strain at section $x$ is directly proportional to the normal force $N(x, \omega)$ at that section and at that frequency, with proportionality constant $1 /(A E(\omega))$. With the state vector defined as in (8), the vector $\mathbf{c}_{0}(\omega)$ in $(6)$ can thus expressed as

$$
\mathbf{c}_{0}(\omega)=\left[\begin{array}{ll}
\frac{1}{A E(\omega)} & 0
\end{array}\right]^{T}
$$

\subsection{Flexural waves}

Now consider the linearly viscoelastic, homogenous beam of length $L$, with cross-sectional area $A$, moment of inertia $I$, and density $\rho$. To describe the flexural wave propagation at section $x$, appropriate choices of state variables are the bending moment $M(x, t)$, the shear force $Q(x, t)$, the rotational velocity $\dot{\phi}(x, t)$, and the time derivative of the center line deflection $\dot{w}(x, t)$. See [9] for further details. This gives the frequency domain state vector

$$
\mathbf{s}(x, \omega)=\left[\begin{array}{llll}
Q(x, \omega) & \dot{w}(x, \omega) & M(x, \omega) & \dot{\phi}(x, \omega)
\end{array}\right]^{T} .
$$

With the Timoshenko ${ }^{1}$ beam theory, the matrix $\mathbf{R}(\omega)$ can be defined as

$$
\mathbf{R}(\omega)=\left[\begin{array}{cccc}
0 & \mathrm{i} \omega \rho A & 0 & 0 \\
\frac{\mathrm{i} \omega \psi}{E(\omega) A} & 0 & 0 & -1 \\
1 & 0 & 0 & \mathrm{i} \omega \rho I \\
0 & 0 & \frac{\mathrm{i} \omega}{I E(\omega)} & 0
\end{array}\right]
$$

\footnotetext{
${ }^{1}$ Another and more simplified theory is the Euler-Bernoulli beam theory, see [17].
} 
Note that we have here introduced the known constant $\psi$, which is dependent on the cross-sectional geometry and the Poisson's ratio of the material, see [9]. The matrix $\mathbf{R}(\omega)$ has four eigenvalues and the general solution (7) will be

$$
\mathbf{s}(x, \omega)=\mathbf{c}_{1}^{-}(\omega) \mathrm{e}^{\gamma_{1}(\omega) x}+\mathbf{c}_{1}^{+}(\omega) \mathrm{e}^{-\gamma_{1}(\omega) x}+\mathbf{c}_{2}^{-}(\omega) \mathrm{e}^{\gamma_{2}(\omega) x}+\mathbf{c}_{2}^{+}(\omega) \mathrm{e}^{-\gamma_{2}(\omega) x} .
$$

In order to determine the wave propagation functions $\{ \pm \gamma(\omega)\}_{i=1}^{2}$ as the eigenvalues of $\mathbf{R}(\omega)$, we need to solve the characteristic equation of $\mathbf{R}(\omega)$, which is given by

$$
\gamma^{4}(\omega)+2 a(\omega) \gamma^{2}(\omega)-b(\omega)=0
$$

where

$$
\begin{gathered}
a(\omega)=\frac{\rho \omega^{2}}{2 E(\omega)}(1+\psi) \\
b(\omega)=\frac{\rho \omega^{2}}{E(\omega)}\left(\frac{A}{I}-\frac{\rho \omega^{2}}{E(\omega)} \psi\right)
\end{gathered}
$$

Hence from (16) the wave propagation functions are given by

$$
\begin{aligned}
& \gamma_{1}^{2}(\omega)=-a(\omega)+\sqrt{a^{2}(\omega)+b(\omega)} \\
& \gamma_{2}^{2}(\omega)=-a(\omega)-\sqrt{a^{2}(\omega)+b(\omega)} .
\end{aligned}
$$

which also describes the relationship between the wave propagation functions and the complex modulus $E(\omega)$.

For flexural waves, the strain at section $x$ is directly proportional to the bending moment at that section and at that frequency with proportionality constant $A /(I E(\omega))$. With the state vector defined as in (13), the vector $\mathbf{c}_{0}(\omega)$ in $(6)$ is thus defined as

$$
\mathbf{c}_{0}(\omega)=\left[\begin{array}{llll}
0 & 0 & \frac{A}{I E(\omega)} & 0
\end{array}\right]^{T} .
$$

\section{Identification}

In this section, we consider the identification of the complex modulus $E(\omega)$ from the sampled strain data at a set of predefined sections $\left\{x_{i}\right\}_{i=1}^{n}$, as shown in Figure 1 and 2. We here assume that the sampled data is corrupted by spatially and temporally white Gaussian noise with zero mean and variance $\lambda$. If we denote the frequency domain noise by $v(x, \omega)$, then the Fourier transform strain measurements can be written as

$$
\varepsilon(x, \omega)=\varepsilon_{0}(x, \omega)+v(x, \omega),
$$


where $\varepsilon_{0}(x, \omega)$ denotes the noise-free strain from section $x$.

Now define the measurement vector $\varepsilon(\omega)$, the noise-free strain vector $\boldsymbol{\varepsilon}_{0}(\omega)$ and the noise vector $\mathbf{v}(\omega)$ as

$$
\begin{aligned}
\boldsymbol{\varepsilon}(\omega) & =\left[\begin{array}{lll}
\varepsilon\left(x_{1}, \omega\right) & \cdots & \varepsilon\left(x_{n}, \omega\right)
\end{array}\right]^{T}, \\
\boldsymbol{\varepsilon}_{0}(\omega) & =\left[\begin{array}{lll}
\varepsilon_{0}\left(x_{1}, \omega\right) & \cdots & \varepsilon_{0}\left(x_{n}, \omega\right)
\end{array}\right]^{T}, \\
\mathbf{v}(\omega) & =\left[\begin{array}{lll}
v\left(x_{1}, \omega\right) & \cdots & v\left(x_{n}, \omega\right)
\end{array}\right]^{T} .
\end{aligned}
$$

By combining (7), (6) and (22) with the definitions in (23) we get

$$
\boldsymbol{\varepsilon}(\omega)=\mathbf{A}(\omega) \mathbf{c}(\omega)+\mathbf{v}(\omega)
$$

where

$$
\mathbf{A}(\omega)=\left[\begin{array}{ccccc}
\mathrm{e}^{\gamma_{1} x_{1}} & \mathrm{e}^{-\gamma_{1} x_{1}} & \cdots & \mathrm{e}^{\gamma_{p} x_{1}} & \mathrm{e}^{-\gamma_{p} x_{1}} \\
\vdots & \vdots & & \vdots & \vdots \\
\mathrm{e}^{\gamma_{1} x_{n}} & \mathrm{e}^{-\gamma_{1} x_{n}} & \cdots & \mathrm{e}^{\gamma_{p} x_{n}} & \mathrm{e}^{-\gamma_{p} x_{n}}
\end{array}\right] .
$$

Note that $\mathbf{A}(\omega)$ will be an $n \times 2$ matrix for longitudinal waves, and an $n \times 4$ matrix for flexural waves. Here, we have also introduced the unknown frequency dependent vector $\mathbf{c}(\omega)$ given by

$$
\mathbf{c}(\omega)=\left[\begin{array}{lllll}
\mathbf{c}_{1}^{-}(\omega) & \mathbf{c}_{1}^{+}(\omega) & \cdots & \mathbf{c}_{p}^{-}(\omega) & \mathbf{c}_{p}^{+}(\omega)
\end{array}\right] \mathbf{c}_{o}(\omega) .
$$

The vectors in (26) are all of dimension $p \times 1$.

In conformity with the notations in [14], we will here introduce the frequency dependent real-valued vector

$$
\mathbf{e}_{\omega}=\left[\begin{array}{ll}
e_{r}(\omega) & e_{i}(\omega)
\end{array}\right]^{T}
$$

such that

$$
E(\omega)=e_{r}(\omega)+\mathrm{i} e_{i}(\omega) .
$$

To stress that the matrix $\mathbf{A}(\omega)$ is an implicit analytic function of the complex modulus, we will also change this notation to $\mathbf{A}\left(\mathbf{e}_{\omega}\right)$. The notation in (27) is helpful since it allows us to deal with a real-valued parameter vector. An estimate $\hat{\mathbf{e}}_{\omega}$ of $\mathbf{e}_{\omega}$ can then be obtained through the solution to the separable nonlinear least squares problem

$$
\hat{\mathbf{e}}_{\omega}=\arg \min _{\mathbf{c}(\omega), \mathbf{e}_{\omega}}\left\|\varepsilon(\omega)-\mathbf{A}\left(\mathbf{e}_{\omega}\right) \mathbf{c}(\omega)\right\|^{2}
$$

where the unknown vector $\mathbf{c}(\omega)$ acts as a nuisance parameter vector of dimension $p$. The number of independent measurements $n$ thus has to satisfy 
$n \geq p+1$. For a detailed description of the numerical issues of this estimation problem, we refer the reader to [14].

In [14] an expression for the covariance matrix for the estimator $\hat{\mathbf{e}}_{\omega}$ is also derived. For any two discrete frequencies $\omega_{k}$ and $\omega_{l}$, and under the assumption that the signal-to-noise ratio is large, we have that

$$
\mathrm{E}\left\{\left(\hat{\mathbf{e}}_{\omega_{l}}-\mathbf{e}_{\omega_{l}}^{0}\right)\left(\hat{\mathbf{e}}_{\omega_{k}}-\mathbf{e}_{\omega_{k}}^{0}\right)^{T}\right\}=\frac{\lambda}{2 h\left(\omega_{l}\right)} \delta_{l, k} \mathbf{I}_{2}, \quad 0<k, l<N / 2
$$

where $\lambda$ is the noise variance, $\delta_{l, k}$ the Kronecker delta function, and $\mathbf{e}_{\omega_{l}}^{0}$ denotes the true parameter vector. Further,

$$
h(\omega)=\boldsymbol{\varepsilon}_{0}^{*}(\omega) \mathbf{A}^{\dagger *}\left(\mathbf{e}_{\omega}^{0}\right) \mathbf{A}_{r}^{*}\left(\mathbf{e}_{w}^{0}\right) \mathbf{P}\left(\mathbf{e}_{\omega}^{0}\right) \mathbf{A}_{r}\left(\mathbf{e}_{\omega}^{0}\right) \mathbf{A}^{\dagger}\left(\mathbf{e}_{\omega}^{0}\right) \boldsymbol{\varepsilon}_{0}(\omega),
$$

where $\mathbf{A}^{\dagger}\left(\mathbf{e}_{\omega}\right)$ is the pseudo-inverse of $\mathbf{A}\left(\mathbf{e}_{\omega}\right), \mathbf{P}\left(\mathbf{e}_{\omega}\right)$ is the orthogonal projection onto the null space of $\mathbf{A}^{*}\left(\mathbf{e}_{\omega}\right)$ given by

$$
\mathbf{P}\left(\mathbf{e}_{\omega}\right)=\mathbf{I}_{n}-\mathbf{A}\left(\mathbf{e}_{\omega}\right) \mathbf{A}^{\dagger}\left(\mathbf{e}_{\omega}\right)
$$

and $\mathbf{A}_{r}\left(\mathbf{e}_{\omega}\right)$ is defined as

$$
\mathbf{A}_{r}\left(\mathbf{e}_{\omega}\right)=\frac{\partial \mathbf{A}\left(\mathbf{e}_{\omega}\right)}{\partial e_{r}(\omega)} .
$$

From the expression (30) for the covariance matrix, we see that the estimate of the complex modulus has the following properties:

- The estimate at a particular frequency is uncorrelated with the estimate at any other frequency.

- The estimates of the real and the imaginary part are uncorrelated at any given frequency.

- The estimates of the real and the imaginary part are of equal variance, proportional to $1 / h(\omega)$.

- The variance, and thereby the estimation accuracy, is implicitly dependent on the sensor locations and the type of excitation.

- The function $h(\omega)$ is directly proportional to the signal energy, and the variance is inversely proportional to the signal-to-noise ratio.

The influence of the sensor placement on the estimation accuracy has been studied in [18] and [21], while the impact of the chosen excitation will be studied in following sections. 


\section{Experiment design}

The common procedure for optimal experiment design is to minimize some scalar function of the covariance matrix $P$ of the estimate. Popular choices of scalar functions include

$$
\begin{array}{ll}
\text { A-optimality : } & \min \operatorname{tr}(P) \\
\text { D-optimality : } & \min \operatorname{det}(P) \\
\text { E-optimality : } & \min \lambda_{\max }(P) .
\end{array}
$$

Note that (34) applies in the case of parametric identification, where there is one covariance matrix for the parameter estimate. Here we are concerned with nonparametric identification, and accordingly we have one covariance matrix for each frequency at which we have identified the complex modulus. For the nonparametric case we are confined to minimizing the covariance matrix on average, by integrating over some frequency interval. Focusing on A- and D-optimality we get

$$
\begin{array}{ll}
\text { A - optimality : } & \min \int_{\Omega} \operatorname{tr}\left(P_{N}(\omega)\right) d \omega \\
\text { D - optimality : } & \min \int_{\Omega} \operatorname{det}\left(P_{N}(\omega)\right) d \omega
\end{array}
$$

where $P_{N}$ is the covariance matrix for the nonparametric estimate, and $\Omega$ is the frequency interval.

In order to do experiment design with respect to the input signal, we first have to find how the covariance matrix (30) depends on the excitation. We can here use the linear property of the system, i.e. that there is a linear dependency between the input excitation signal $u(\omega)$, and the measured strains

$$
\boldsymbol{\varepsilon}(\omega)=\mathbf{g}(\omega) u(\omega)+\mathbf{v}(\omega) .
$$

By the use of (37), the expression for the covariance matrix in (30) can be reformulated as

$$
P_{N}=\frac{\lambda}{2\left|u\left(\omega_{l}\right)\right|^{2} l\left(\omega_{l}\right)} \delta_{l, k} \mathbf{I}_{2}, \quad 0<k, l<N / 2
$$

where

$$
l(\omega)=\mathbf{g}^{*}(\omega) \mathbf{A}^{\dagger *}\left(\mathbf{e}_{\omega}^{0}\right) \mathbf{A}_{r}^{*}\left(\mathbf{e}_{w}^{0}\right) \mathbf{P}\left(\mathbf{e}_{\omega}^{0}\right) \mathbf{A}_{r}\left(\mathbf{e}_{\omega}^{0}\right) \mathbf{A}^{\dagger}\left(\mathbf{e}_{\omega}^{0}\right) \mathbf{g}(\omega) .
$$

The function $l(\omega)$ depends solely on the material characteristics and the sensor positions. How to find the function $\mathbf{g}(\omega)$ used in (39) is described in 
Appendix B. Note that when $\mathbf{g}(\omega)$ has been found, the input signal used in a particular experiment can easily be identified from the measured strains, by the use of (37). Also note that depending on how the experiment is set up, the input for flexural waves can be either the shear force $Q$, or the rotational velocity $\dot{w}$, at the point of excitation. For longitudinal waves, the input is the normal force $N$ at the point of excitation.

As the covariance matrix is inversely proportional to the signal energy, it is obvious that we can make the variance arbitrarily small by choosing $|u(\omega)|^{2}$ large, see (38). To keep the input energy on a reasonable level, we must therefore constrain it in the optimizations. This will give the following problem, which can be solved by calculus of variations, see [5] or [22].

Optimal experiment design: Let the criterion function be

$$
V=\int_{\Omega} \frac{1}{\left(|u(\omega)|^{2} l(\omega)\right)^{p}} d \omega, \quad \Omega=\left[\omega_{1}, \omega_{2}\right] .
$$

For A-optimality choose $p=1$, and for D-optimality choose $p=2$. The optimization problem can then be formulated as

$$
\min _{|u(\omega)|^{2}} V \quad \text { s.t. } \quad \int_{\Omega}|u(\omega)|^{2} d \omega=\alpha .
$$

The solution to the optimization problem in (41) is given by

$$
\left|u_{o p t}(\omega)\right|^{2}=\frac{1}{(l(\omega))^{p /(p+1)}} \frac{\alpha}{\int_{\Omega} \frac{1}{(l(\omega))^{p /(p+1)}}} d \omega .
$$

The optimal value is given by

$$
V_{\text {opt }}=\frac{1}{\alpha^{p}}\left(\int_{\Omega} \frac{1}{(l(\omega))^{p /(p+1)}} d \omega\right)^{p+1} .
$$

For a proof, see Appendix C.

Remark 1: Since it is trivially so that we want to use all the input energy avaliable, we have here chosen the constraint on input energy in (41) as an equality rather than an inequality.

Remark 2: Since the input energy outside the the interval $\Omega$ will have no effect on the estimates within that interval, the input energy is set to zero for frequencies outside $\Omega$. 


\section{Investigations}

The following investigations are based on data taken from live experiments. In the longitudinal case, a PMMA (plexiglass) bar specimen of length $L=2$ $\mathrm{m}$ and diameter $d=16 \mathrm{~mm}$ was used. A discussed in Section 2, this gives a range of useful frequencies of approximately $200 \mathrm{~Hz}-14 \mathrm{kHz}$. The sensor positions $\left\{x_{i}\right\}_{i=1}^{n}$ used in the experiment was

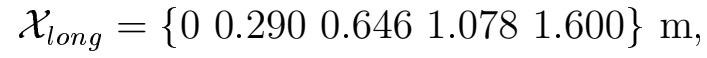

and the number of data points collected from each sensor $N=2^{12}$ at a sampling interval of $T=20 \mu \mathrm{s}$. The density of PMMA is $\rho=1183 \mathrm{~kg} / \mathrm{m}^{3}$. Additional information on these experiments can be found in [10].

No experiments have yet been made on the experimental setup for flexural waves in Figure 2. However, to evaluate the performance of the optimal excitation, data from a similar experiment, described in [15], has been used. The drawback with this data is that the boundary conditions in Appendix A are not fulfilled at $x=0$, which means that the the excitation used in a particular experiment can not be identified.

The optimal excitation for flexural waves was evaluated for the same experimental conditions as in [15]. There, a beam made of PP (polypropylene), with density $\rho=915 \mathrm{~kg} / \mathrm{m}^{3}$ and Poisson's ratio $\nu=0.33$, was used. The length of the beam was $L=1.5 \mathrm{~m}$, and the sensor positions $\left\{x_{i}\right\}_{i=1}^{n}$

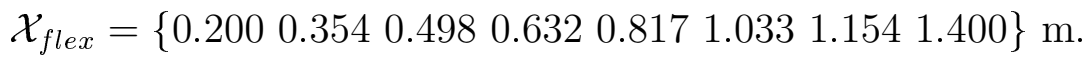

The number of data points collected from each sensor was $N=2^{16}$ at a sampling interval of $T=50 \mu \mathrm{s}$. For more information, see [15]. As discussed in Section 2, flexural wave experiments are useful for frequencies between 10 $\mathrm{Hz}$ and $1 \mathrm{kHz}$. Due to the numerical difficulties mentioned in Appendix B, this investigation has to be confined to frequencies below $550 \mathrm{~Hz}$.

The problem can now be formulated as follows. In both the longitudinal and the flexural wave experiment described above, a pulse generated by the impact of a steel hammer or pendulum was used as an input. This pulse has a certain energy content in the useful frequency range, and the energy is distributed over the frequencies in a certain way. If we instead could distribute this energy freely over the useful frequencies, what would this distribution optimally be? This problem formulation gives rise several interesting questions, which will be discussed in the following. For the longitudinal wave experiments, the energy content of the original pulse is easily estimated by first identifying the excitation used in the experiment. For the flexural wave data, the original exciting pulse and its energy content can not be known exactly, as discussed above. We instead have to guess the input power $\alpha$ used 


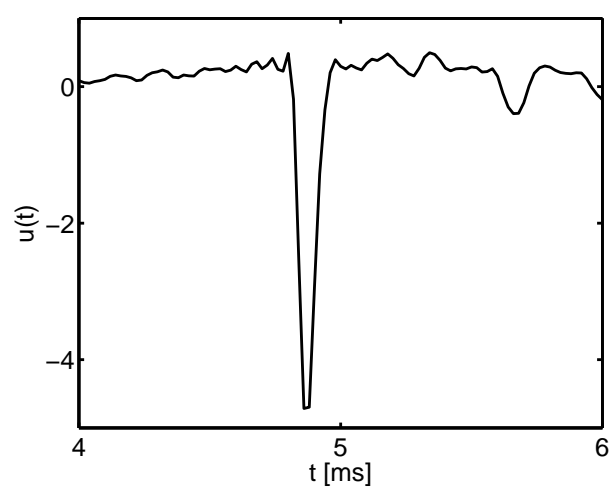

(a)

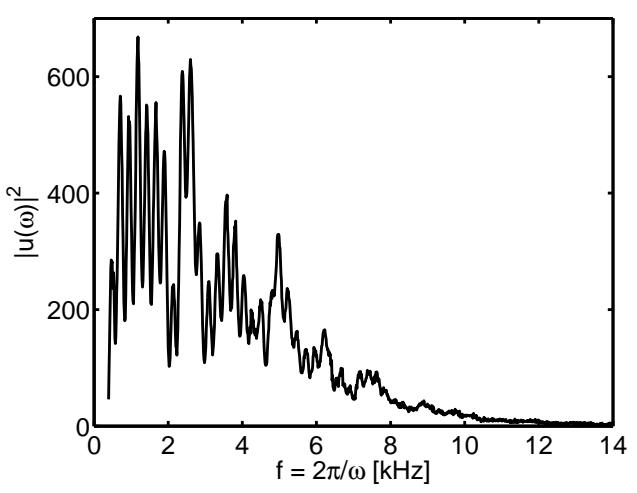

(b)

Figure 3: Typical input signal for a longitudinal wave experiment (a), and its spectrum (b).

in the optimal experiment design in (41). However, since $\alpha$ enters only as a scaling factor in the optimal spectrum (42), this guess will have no influence on how the power is distributed over the frequencies. Note that this is a theoretical investigation, and that no consideration has been taken as to the implementability of the achieved input spectra. This is a question for future work.

Example 1: What input signal has been used in a particular experiment?

In the experiments described above, an unknown pulse generated through impact with a steel hammer, was used as an input signal. For the longitudinal wave experiment, this pulse can be identified through (62) and the measured strain data. A typical input signal for the experiment is shown in Figure 3, along with its power spectra. As can be seen, the impulse has most of its energy concentrated at low frequencies; the majority of the power lies below $6 \mathrm{kHz}$.

Example 2: What is the frequency content of an optimal input signal?

In order to get good estimates for all useful frequencies, we want an input signal that excites the system for all the frequencies within that range. For the input signal in Figure 3, it is reasonable to think that the system is insufficiently excited at higher frequencies, since the input lacks power in the higher frequency range. The lower accuracy in the estimates is evident in Figure 4a, where a gradual increase in standard deviations can be noted for frequencies over $6 \mathrm{kHz}$. If the same reasoning is applied to the standard devi- 


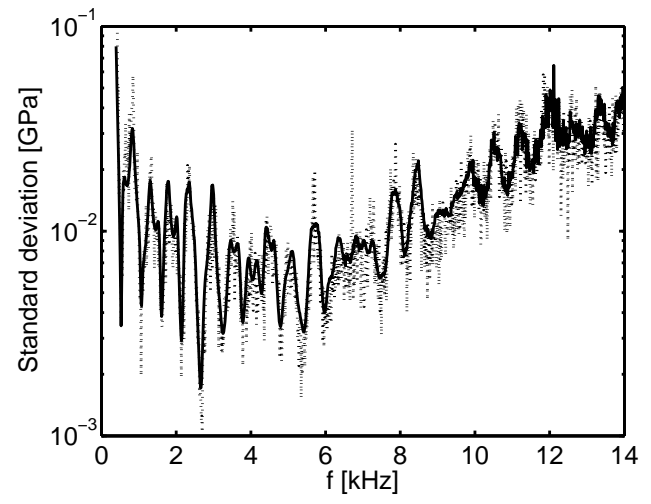

(a)

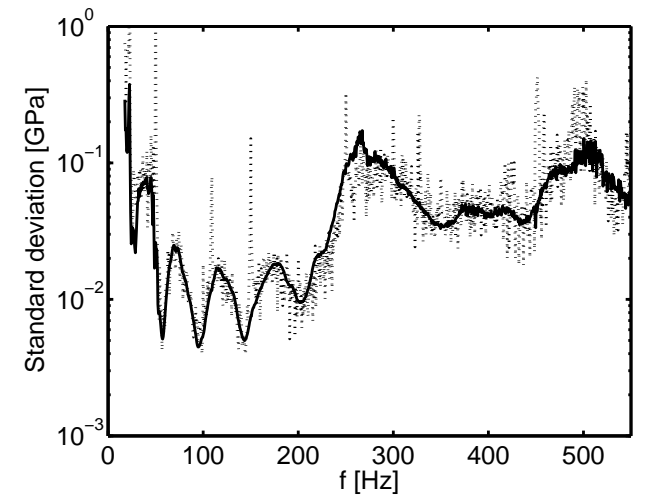

(b)

Figure 4: Standard deviations of the estimated complex modulus, based on experiments using unknown pulse excitation. Longitudinal wave experiments (a), and flexural wave experiments (b). Analytical standard deviation (solid), and experimental standard deviation, 10 independent experiments (dotted).

ations in the flexural case (Figure 4b), one may expect the exciting pulse to have the majority of its power below $250 \mathrm{~Hz}$. The values of the noise variance used for the calculation of the standard deviations, was $\lambda=2.1 \times 10^{-14}$ for longitudinal waves and $\lambda=1.7 \times 10^{-13}$ for flexural waves, see [19] and [15]. The true complex modulus needed to calculate the standard deviations was here replaced by the estimated complex modulus, and the true strains by the measured strains.

The optimal input spectra achieved by (42) are shown in Figure 5. We have here included all the useful frequencies in the minimization, i.e. $\Omega=[200 \mathrm{~Hz}, 14 \mathrm{kHz}]$ for longitudinal waves, and $\Omega=[10 \mathrm{~Hz}, 550 \mathrm{~Hz}]$ for flexural waves. It can be seen that the A- and the D-optimal design give similar results. However, an important advantage of D-optimality is that it is invariant under scale changes in the parameters and linear transformations of the output. Another advantage of D-optimality is that it implies G-optimality, which means that the variance of the predicted output is minimized. These properties have made it one of the most popular methods in optimal experiment design.

From Figure 5, it is also evident that it is better to distribute the energy more evenly over the frequencies; for the longitudinal wave experiment more power has been allocated to the higher frequency range, and a considerable amount of the input power is concentrated to the higher frequency range for 


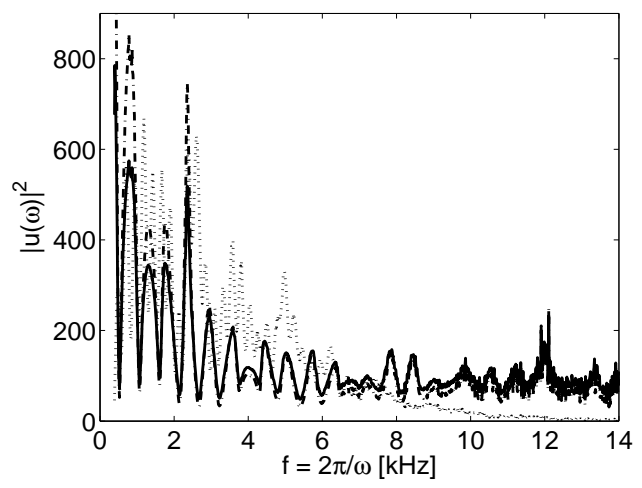

(a)

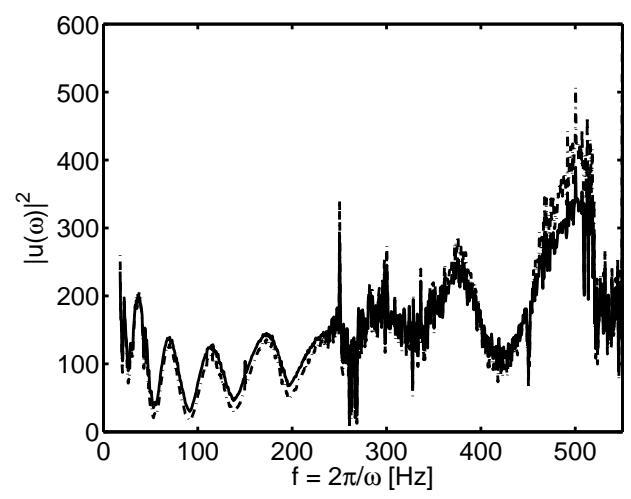

(b)

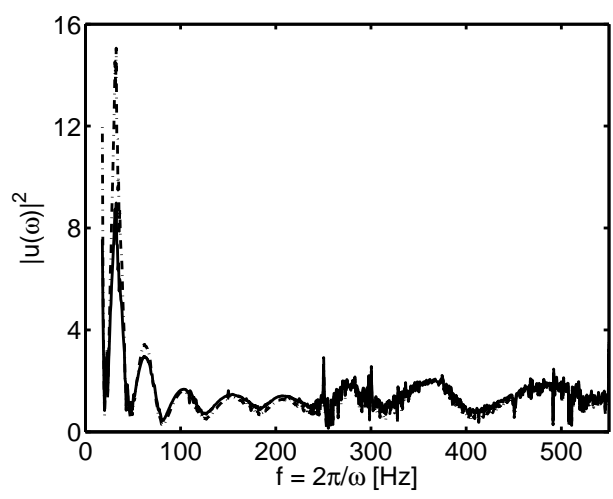

(c)

Figure 5: Optimal input spectra. Longitudinal wave experiments in (a), and flexural wave experiments in (b)-(c). In (b) $u(\omega)=Q(0, \omega)$, and in (c) $u(\omega)=\dot{w}(0, \omega)$. A-optimal input (solid), and D-optimal input (dash-dotted). In (a), identified pulse excitation (dotted).

flexural waves. It is also interesting to compare the optimal input spectra in Figure 5 to the standard deviations in Figure 4. Clearly, the procedure allocates more input energy to those frequencies for which the current sensor position yields a poor estimate, i.e. where the standard deviations are increased. Intuitively it makes sense that areas of increased variance needs to be suppressed when minimizing a criterion function like the one in (40). This is done by extracting more information from these modes, here achieved 


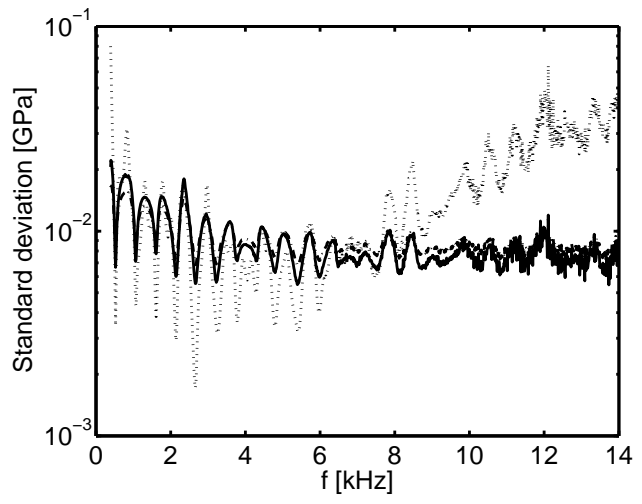

(a)

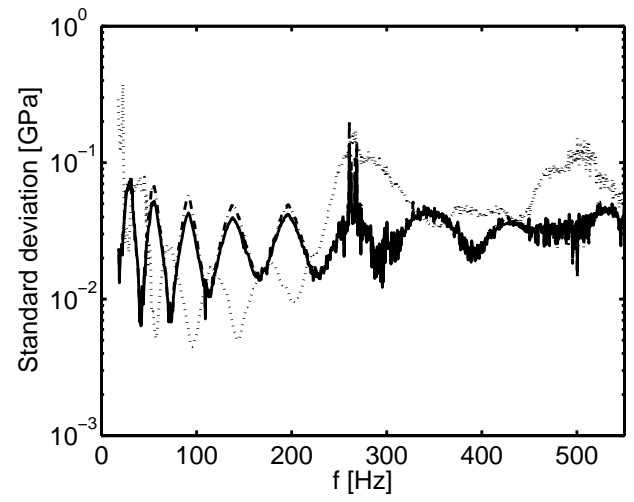

(b)

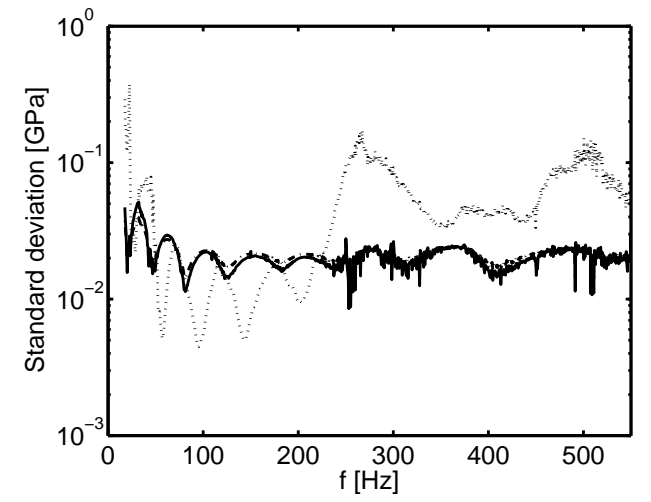

(c)

Figure 6: Standard deviations. Longitudinal wave experiments (a), and flexural wave experiments (b)-(c). In (b) $u(\omega)=Q(0, \omega)$, and in (c) $u(\omega)=\dot{w}(0, \omega)$. Original impulse excitation (dotted), A-optimal input (solid), and D-optimal input (dash-dotted).

by allocating more input power around certain frequencies, and thereby get a better estimate.

Example 3: What can be gained in estimation accuracy with the use of an optimal input signal?

From Figure 6 it is clear that it is possible to achieve better estimate by using an optimal distribution of input power, compared to that of the classical 


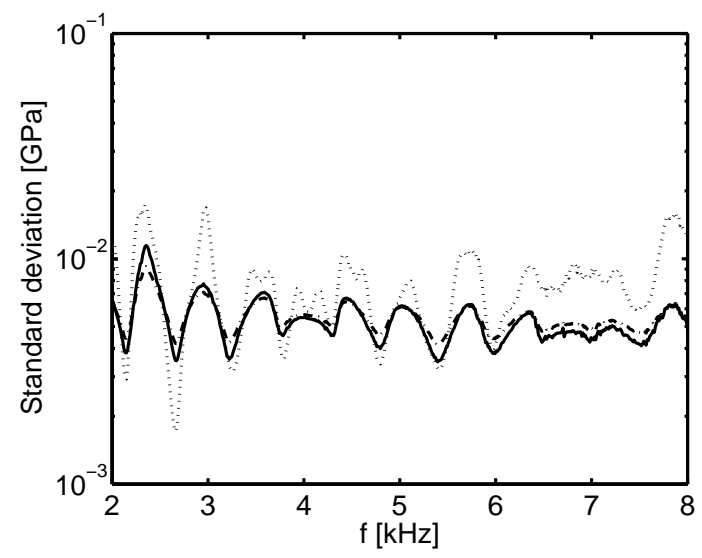

Figure 7: Standard deviations for a longitudinal wave experiment with optimal input in the range $2-8 \mathrm{kHz}$. Identified impulse excitation (dotted), A-optimal input (solid), and D-optimal input (dash-dotted).

impact excitation. For longitudinal wave experiments, the average standard deviation has decreased by around $40 \%$ over the range of useful frequencies. The corresponding decrease in maximal standard deviation is about $70 \%$. No such comparison can be made for flexural waves, since we do not know the energy content of the original excitation signal. As was seen in Section 4, the accuracy of the estimates are inversely proportional to the signal energy, and comparing experiments with different energy contents may therefore be deceiving. It is however clear that for both flexural and longitudinal waves, the optimal input will counteract the increase in standard deviation in the high frequency range. Instead the accuracy is kept on approximately the same level for all frequencies. The input power $\alpha$ for the flexural wave experiment has here been chosen based on the results from the longitudinal wave case, i.e. so that the standard deviations for the optimal input lies between the higher and the lower values of the original standard deviations.

From Figure 6, it is also clear that keeping standard deviations on the same level for all frequencies tends to decrease standard deviations in the higher frequency range, at the expense of accuracy at lower frequencies. It is however possible to target specific areas and get better accuracy at frequencies with already low variance. In Figure 7 , the range $2-8 \mathrm{kHz}$ was targeted for a longitudinal wave experiment, with lower standard deviations as a result. 


\section{Conclusions}

This paper treats the problem of optimal excitation for estimating the complex modulus of a viscoelastic material from wave propagation experiments. In this study, we focus on nonparametric identification in the frequency domain. It is important to stress that even though this article treats a specific application, the procedure for optimal experiment design used here also applies to the more general case of identification experiments. The key issue is to have a covariance matrix dependent on the design parameters of interest.

Here, the idea is that if we can distribute the energy content of the input excitation signal in an optimal way, it is possible to maximize the amount of useful information that can be extracted from the data, and thereby get a more accurate estimate. The typical way to excite the system in this kind of experiments has been through impact by the use of a steel hammer or an air gun; a kind of excitation that has most of its power in the lower frequency range. It was found that more accurate estimates can be achieved by spreading the input power more evenly over the frequencies, and that it is advantageous to allocate more energy to frequencies where the estimates are particularly bad. It was also shown how the theory can be used to identify the excitation used in a particular experiment, since this is generally not known and hard to measure. Other relevant design parameters for this application is the number of sensors used in the experiment, and their locations. This problem was studied in [18] and [21].

In order to confirm the results of this paper, experiments have to be designed where the optimal input signal can be applied to the system. Issues to consider in this work include:

- What is the effect of the equipment used? This includes the transfer functions of the amplifier and the shaker used to apply force to the specimen. These need to be compensated for to some extent. Specifically, there will be an upper limit on how fast the shaker can work, and thereby how high frequencies that can be generated.

- How much energy can we drive into the specimen? This issue concerns the time span of the experiment, since more energy is generated the longer the experiment continues.

\section{Acknowledgment}

This research was partially supported by the Swedish Research Council, contract 621-2004-5169. The authors also want to thank Dr. Lars Hillström and Mr. Saed Mousavi for providing the data used in this study. 


\section{A Boundary conditions}

\section{A.1 Longitudinal waves}

If the longitudinal wave propagation experiment in Figure 1 is designed so that there is a free end at $x=L$, the strain (and thereby the normal force $N)$ at that section will equal zero. The input signal to the system is the normal force at $x=0$ (the point of excitation), which gives the following set of boundary conditions

$$
N(0, \omega)=u(\omega), \quad N(L, \omega)=0 .
$$

\section{A.2 Flexural waves}

For a flexural wave propagation experiment designed as in Figure 2, with a free end at $x=L$, it holds that the shear force $Q$ equals zero at $x=L$. Moreover, the bending moment $M$ equals zero both at $x=L$ and at $x=0$. Depending on the experimental setup, the input signal to the system can be either the shear force $Q$ or the rotational velocity $\dot{w}$ at the point of excitation $(x=0)$, which gives the following set of boundary conditions

$$
Q(0, \omega) \text { or } \dot{w}(0, \omega)=u(\omega), M(0, \omega)=0, Q(L, \omega)=0, M(L, \omega)=0 .
$$

\section{B Derivation of $\mathbf{g}(\omega)$}

First note that the general solution of the system of difference equations in (5) can also be written on the form

$$
\mathbf{s}(x, \omega)=\mathrm{e}^{\mathbf{R}(\omega) x} \mathbf{s}(0, \omega) .
$$

Combining (48) with (6), (22) and the definitions in (23) we get

$$
\boldsymbol{\varepsilon}(\omega)=\Gamma(\omega) \mathbf{s}(0, \omega)+\mathbf{v}(\omega)
$$

where

$$
\Gamma(\omega)=\left[\begin{array}{c}
\mathbf{c}_{0}^{T}(\omega) \mathrm{e}^{\mathbf{R}(\omega) x_{1}} \\
\vdots \\
\mathbf{c}_{0}^{T}(\omega) \mathrm{e}^{\mathbf{R}(\omega) x_{n}}
\end{array}\right] .
$$

To find the relationship between the input signal and $\mathbf{s}(0, \omega)$, we can apply the boundary conditions described in Appendix A. These give

$$
\begin{aligned}
& B_{1} \mathbf{s}(0, \omega)=\mathbf{e}_{1} u(\omega), \\
& B_{2} \mathbf{s}(L, \omega)=\mathbf{0}
\end{aligned}
$$


with $B_{1}, B_{2}$ and $\mathbf{e}_{1}$ defined as

$$
B_{1}=\left[\begin{array}{ll}
1 & 0
\end{array}\right], \quad B_{2}=\left[\begin{array}{ll}
1 & 0
\end{array}\right], \quad \mathbf{e}_{1}=1
$$

for longitudinal waves. For flexural waves $B_{1}$ depends on the choice of input signal,

$$
B_{1}=\left[\begin{array}{llll}
1 & 0 & 0 & 0 \\
0 & 0 & 0 & 1
\end{array}\right] \text { for } u(\omega)=Q(0, \omega)
$$

or

$$
B_{1}=\left[\begin{array}{llll}
0 & 1 & 0 & 0 \\
0 & 0 & 0 & 1
\end{array}\right] \text { for } u(\omega)=\dot{w}(0, \omega) \text {, }
$$

while

$$
B_{2}=\left[\begin{array}{llll}
1 & 0 & 0 & 0 \\
0 & 0 & 1 & 0
\end{array}\right], \quad \mathbf{e}_{1}=\left[\begin{array}{ll}
1 & 0
\end{array}\right]^{T} .
$$

Together, (51) and (52) can be written as

$$
\Lambda(\omega) \mathbf{s}(0, \omega)=\mathbf{e} u(\omega)
$$

with

$$
\Lambda(\omega)=\left[\begin{array}{c}
B_{1} \\
B_{2} \mathrm{e}^{\mathbf{R}(\omega) L}
\end{array}\right]
$$

and

$$
\mathbf{e}=\left[\begin{array}{ll}
\mathbf{e}_{1}^{T} & \mathbf{0}^{T}
\end{array}\right]^{T} .
$$

Combining (57) with (49) then gives

$$
\boldsymbol{\varepsilon}(\omega)=\Gamma(\omega) \Lambda^{-1}(\omega) \mathbf{e} u(\omega)+\mathbf{v}(\omega),
$$

and the function $\mathbf{g}(\omega)$ given in (37) satisfies

$$
\mathbf{g}(\omega)=\Gamma(\omega) \Lambda^{-1}(\omega) \mathbf{e}
$$

Note that we have to assume the matrix $\Lambda(\omega)$ to be invertible for all frequencies. This assumption is easily shown to be fulfilled for longitudinal waves, while ill-conditioned matrices may occur for flexural waves. Also note that the function $\mathbf{g}(\omega)$ can be used to identify the input in a particular experiment from the measured strain data. This can be done in a least squares sense by applying

$$
u(\omega)=\mathbf{g}^{\dagger}(\omega) \boldsymbol{\varepsilon}(\omega),
$$

where $\mathbf{g}^{\dagger}(\omega)$ denotes the pseudo-inverse of $\mathbf{g}(\omega)$. 


\section{Solution to the optimization problem}

A problem on the form

$$
\min _{y(\omega)} \int_{\omega_{1}}^{\omega_{2}} f\left(\omega, y(\omega), y^{\prime}(\omega)\right) d \omega
$$

can be solved by calculus of variations, see [5] or [22]. According to this theory, the minimizing function of $(63), y^{*}(\omega)$, must satisfy the Euler equation

$$
\frac{d}{d \omega}\left(\frac{\partial f}{\partial y^{\prime}}\right)-\frac{\partial f}{\partial y}=0
$$

where the indices of the functions have been omitted for brevity. The closely related problem with an integral side condition

$$
\min _{y(\omega)} \int_{\omega_{1}}^{\omega_{2}} f\left(\omega, y(\omega), y^{\prime}(\omega)\right) d \omega \quad \text { s.t. } \int_{\omega_{1}}^{\omega_{2}} g\left(\omega, y(\omega), y^{\prime}(\omega)\right) d \omega=\alpha
$$

can be handled in the same way by first introducing the Lagrange multiplier $\lambda$, see [2]. The minimum of (65) can then be found through solving the equivalent optimization problem

$$
\min _{y(\omega)} \int_{\omega_{1}}^{x_{2}} F\left(\omega, y(\omega), y^{\prime}(\omega)\right) d \omega
$$

where

$$
F\left(\omega, y(\omega), y^{\prime}(\omega)\right)=f\left(\omega, y(\omega), y^{\prime}(\omega)\right)+\lambda g\left(\omega, y(\omega), y^{\prime}(\omega)\right) .
$$

Note that $f(\cdot)$ will now be replaced by $F(\cdot)$ in $(64)$.

For the design problem in (40) and (41), we have $y(\omega)=|u(\omega)|^{2}$. This gives

$$
\begin{aligned}
f\left(\omega, y(\omega), y^{\prime}(\omega)\right) & =\frac{1}{(y(\omega) l(\omega))^{p}} \\
g\left(\omega, y(\omega), y^{\prime}(\omega)\right) & =y(\omega) \\
F\left(\omega, y(\omega), y^{\prime}(\omega)\right) & =\frac{1}{(y(\omega) l(\omega))^{p}}+\lambda y(\omega)
\end{aligned}
$$

Since $F(\cdot)$ does not include $y^{\prime}(\omega)$, the Euler equation for this problem is reduced to

$$
\frac{\partial F}{\partial y}=-\frac{p}{(y(\omega))^{p+1}(l(\omega))^{p}}+\lambda=0 .
$$


The minimizing function $y^{*}(\omega)$ thus has to satisfy

$$
y^{*}(\omega)=\left(\frac{p}{\lambda(l(\omega))^{p}}\right)^{1 /(p+1)} .
$$

To find the value of the Lagrange multiplier $\lambda$ at the optimum, we can use the constraint in $(65)$. With $g(\cdot)$ defined as in (68), at the minimum point we have

$$
\int_{\omega_{1}}^{\omega_{2}} y^{*}(\omega) d \omega=\frac{1}{\lambda^{1 /(p+1)}} \int_{\omega_{1}}^{\omega_{2}}\left(\frac{p}{(l(\omega))^{p}}\right)^{1 /(p+1)} d \omega=\alpha
$$

which gives

$$
\lambda=\left(\frac{1}{\alpha} \int_{\omega_{1}}^{\omega_{2}}\left(\frac{p}{(l(\omega))^{p}}\right)^{1 /(p+1)} d \omega\right)^{p+1} .
$$

Substituting $\lambda$ into (70), we get the minimizing function

$$
y^{*}(\omega)=\left|u_{o p t}(\omega)\right|^{2}=\frac{1}{(l(\omega))^{p /(p+1)}} \frac{\alpha}{\int_{\Omega} \frac{1}{(l(\omega))^{p /(p+1)}}} d \omega,
$$

which equals (42). The optimal function value in (43) follows from straightforward application of (42) in (40).

\section{References}

[1] R. H. Blanc. Transient wave propagation methods for determining the viscoelastic properties of solids. Journal of Applied Mechanics, 60:763$768,1993$.

[2] S. Boyd and L. Vandenberge. Convex Optimization. Cambridge University Press, Cambridge, 2004.

[3] V. V. Fedorov. Theory of Optimal Experiments. Academic Press, New York, 1972.

[4] U. Forsell and L. Ljung. Some results on optimal experiment design. Automatica, 36(5):749-756, May 2000.

[5] I. M. Gelfand and S. V. Fomin. Calculus of Variations. Prentice-Hall, Englewood Cliffs, N.J., 1963.

[6] M. Gevers and X. Bombois. Input design: From open-loop to controloriented design. In Proc. 14th IFAC Symp. System Identification, pages 1329-1334, Newcastle, Australia, March 29-31 2006. 
[7] M. Gevers and L. Ljung. Optimal experiment designs with respect to the intended model application. Automatica, 22(5):543-554, 1986.

[8] M. Hawkes and A. Nehorai. Effects of sensor placement on acoustic vector-sensor array performance. IEEE Journal of Oceanic Engineering, 24(1):33-40, January 1999.

[9] L. Hillström and B. Lundberg. Analysis of elastic flexural waves in nonuniform beams based of measurement of strains and accelerations. Journal of Sound and Vibration, 247(2):227-242, October 2001.

[10] L. Hillström, M. Mossberg, and B. Lundberg. Identification of complex modulus from measured strains on an axially impacted bar using least squares. Journal of Sound and Vibration, 230(3):689-707, February 2000 .

[11] H. Hjalmarsson, M. Gevers, and F. De Bruyne. For model-based control design, closed-loop identification gives better performance. Automatica, 32(12):1659-1673, December 1996.

[12] C. S. Kubrusly and H. Malebranche. Sensors and controllers location in distributed systems - a survey. Automatica, 21(2):117-128, 1985.

[13] E. Lizama and D. Šurdilović. Designing G-optimal experiments for robot dynamics identification. In Proc. Int. Conf. on Robotics and Automation, pages 311-316, Minneapolis, MN, USA, April 1996.

[14] K. Mahata, S. Mousavi, T. Söderström, M. Mossberg, U. Valdek, and L. Hillström. On the use of flexural wave propagation experiments for identification of complex modulus. IEEE Trans. on Control Systems Technology, 11(6):863-874, November 2003.

[15] K. Mahata, S. Mousavi, T. Söderström, and U. Valdek. Using boundary conditions for estimation of complex modulus from flexural wave experiments. IEEE Trans. on Control Systems Technology, 13(6):1093-1099, November 2005.

[16] R. K. Mehra. Optimal input signals for parameter estimation in dynamic systems - survey and new results. IEEE Trans. on Automatic Control, 19(6):753-768, 1974.

[17] L. Meirovitch. Principles and Techniques of Vibrations. Prentice-Hall, New York, 1997. 
[18] M. Mossberg. Optimal experimental design for identification of viscoelastic materials. IEEE Trans. on Control Systems Technology, 12(4):578-582, July 2004.

[19] M. Mossberg, L. Hillström, and T. Söderström. Non-parametric identification of viscoelastic materials from wave propagation experiments. Automatica, 37(4):511-521, April 2001.

[20] F. Pukelsheim. Optimal Design of Experiments. Wiley, New York, 1993.

[21] A. Runqvist, M. Mossberg, , and T. Söderström. Optimal sensor locations for nonparametric identification of viscoelastic materials. In Proc. 16th IFAC World Congress, Prague, Czech Republic, July 4-8 2005.

[22] G. F. Simmons. Differential Equations with Applications and Historical Notes. McGraw-Hill, Singapore, 2:nd edition, 1991.

[23] Y. Sogabe and M. Tsuzuki. Identification of the dynamic properties of linear viscoelastic materials by wave propagation testing. Bulletin of the Japan Society of Mechanical Engineers, 29(254):2410-2417, August 1986.

[24] T. Söderström. System identification techniques for estimating material functions from wave propagation experiments. Inverse Problems in Engineering, 10(5):413-439, 2002.

[25] B. Wahlberg, H. Hjalmarsson, and M. Barenthin. On opitmal input design in system identification. In Proc. 14th IFAC Symp. System Identification, pages 499-504, Newcastle, Australia, March 29-31 2006. 